\title{
Vertical Differentiation with Variety-Seeking Consumers
}

\author{
Robert Zeithammer, Raphael Thomadsen \\ Anderson School of Management, University of California, Los Angeles, Los Angeles, California 90095 \\ \{rzeitham@ucla.edu, raphael.thomadsen@anderson.ucla.edu\}
}

\begin{abstract}
$\mathrm{W}^{\mathrm{e}}$ analyze price and quality competition in a vertically differentiated duopoly in which consumers have a preference for variety. The preference for variety is a consequence of diminishing marginal utility for repeated experiences with the same product. We find consumer variety seeking can either soften or intensify price competition, depending on the difference in firm qualities and the strength of consumer preference for variety. When the qualities are similar (or the consumer preference for variety is strong), prices and profits are higher than would be obtained in the absence of variety seeking. On the other hand, if qualities differ enough (or the preference for variety is weak), stronger preferences for variety are associated with more intense price competition and lower profits. When firms set their qualities before competing on price and the range of feasible qualities is restricted such that variety seeking softens competition, competing firms choose to minimally differentiate themselves from each other. The preference for variety can drive the firms to offer multiunit discounts, and the greater price flexibility from these discounts does not necessarily reduce profits relative to simple unit pricing.
\end{abstract}

Key words: marketing; competitive strategy; pricing

History: Received August 3, 2011; accepted April 6, 2012, by J. Miguel Villas-Boas, marketing. Published online in Articles in Advance.

\section{Introduction}

In several product categories, consumers care about both quality and the ability to sample different experiences over multiple consumption occasions. For example, skiers enjoy going to multiple resorts during a single trip to the mountains, even when some of the resorts are objectively better in terms of size, service, and the quality of the runs. Art lovers on a weekend trip to New York may all prefer the larger Metropolitan Museum of Art when they visit town for a single day, but most would also head to another museum if they visited for two weekend days even though seeing all of the art in the Met in a single day is difficult. Finally, suppose you win a weekend of free dinners in Paris. If you care about quality, you surely wish to visit the top-rated restaurant in the city on one of your nights, but you would probably like to try another restaurant (by definition, lower quality) on the second night rather than go to the top-rated one again. A rich literature documents variety-seeking behavior and examines its antecedents (Givon 1984, McAlister and Pessemier 1982, Kahn et al. 1986, McAlister 1982). In the experience-good settings we consider, consumers seek variety because varied experiences provide stimulation and reduce boredom (Faison 1977), satisfy innate human curiosity (Raju 1980), and improve retrospective evaluation of the bundled multiple experiences (Ratner et al. 1999). Note that consumers do not tire of one quality level and want to experience another. Instead, they feel a diminishing marginal utility when they consume multiple identical experiences; choosing variety helps them avoid the diminished utility. ${ }^{1}$

In this paper, we examine how such consumer preferences for variety affect competition between two vertically differentiated firms. In addition to a different quality level, each firm provides a distinct experience and produces multiple units of its product. Consumers buy two units in the category and vary in their willingness to pay for quality. When they consume one unit from each firm, they obtain utility in a manner that is standard in vertically differentiated models. On the other hand, if a consumer buys two units from the same firm, she experiences less utility from the second unit than from the first because of her preference for a variety of experiences. The diminished marginal utility increases the desirability of choosing variety, that is, buying one unit from each

\footnotetext{
${ }^{1}$ The diminishing marginal utility can also arise from satiation on incidental features specific to the each firm's product (McAlister 1982, Feinberg et al. 1992, Trivedi et al. 1994). Villas-Boas (2004) demonstrates that variety-seeking behavior could also arise from a negative switching cost.
} 
firm, relative to a counterfactual world without preferences for variety.

We offer three findings about how preferences for variety affect price and quality competition between the two firms: First, the preference for variety can either intensify or soften price competition. Second, the preference for variety sometimes results in both firms offering the same quality. Third, the preference for variety can drive the firms to offer multiunit discounts, although allowing both firms to use such discounts does not necessarily intensify price competition; it may soften it instead. In the rest of the introduction, we explain the intuition behind each finding and relate it to the literature.

Intuitively, one might expect a consumer preference for variety to always soften price competition: Consider the problem of an avid weekend skier planning a visit to a mountain town near two competing ski resorts that offer similar qualities. Without preferences for variety, the firms would be selling similar products, which would lead to low margins due to intense price competition arising from most skiers choosing the cheaper resort. However, with a preference for variety, avid skiers will choose to ski at each of the resorts once even if one resort's prices are slightly higher than the other's. Therefore, consumer variety seeking reduces downward competitive pressure on prices. Seetharaman and Che (2009) reach a similar conclusion in a two-period horizontally differentiated model in which consumers experience an additional "staying cost" if they consume the same good for both periods. Our first main finding is that this intuition only works when the qualities of the two resorts are similar enough; counterintuitively, consumer preferences for variety can intensify price competition when they are dissimilar.

When the quality difference between the two firms is big enough, for example, when the inferior ski resort has a much lower quality than the superior resort because of its low elevation or diminished snowfall, the cheaper inferior resort attracts some quality-insensitive consumers for both days. In contrast to the situation with similar qualities, the avid skiers now ski at the better resort for the entire weekend, and variety seeking characterizes the behavior of consumers with an intermediate willingness to pay for quality. These intermediate consumers get enough utility from the first day at the better resort to pay for its higher quality, but the discounted utility of the second day is less than the utility they obtain from the other inferior yet cheaper resort. Each firm thus ends up competing to sell both its first and second units, and price competition becomes more intense than if consumers did not prefer variety. Intuitively, competition emerges between the first unit of the inferior firm and the second unit of the superior firm. Because preferences for variety diminish the effective quality of the second superior unit, it is less differentiated from the first unit of the inferior firm, thus intensifying competition.

Given the close relationship between the amount of vertical differentiation and the effect of preferences for variety on price competition, a question arises about the extent to which the two resorts wish to be vertically differentiated. Should an inferior resort remain differentiated or should it improve its quality to be more like the superior resort? In our second main finding, we show that when the range of qualities available to firms is narrow enough, both resorts offer the same quality in equilibrium. This result contrasts with Feinberg et al. (1992), who pioneered research on the supply-side implications of consumer variety seeking and found that firms should always differentiate from each other in the sense that they should increase the value of their unique features and position away from rivals. However, their model is not an equilibrium model and does not consider price competition. Our results also contrast with the basic equilibrium result in vertically differentiated markets without a taste for variety, which shows that firms differentiate themselves to soften price competition (Shaked and Sutton 1982, Moorthy 1988). The intuition behind our result is that variety seeking can soften competition more when the two firms' products are less differentiated, and this softening is stronger than the softening firms can obtain from differentiation. In a separate proposition, we show that the softening of price competition due to consumer preferences for variety is so strong that not only does the inferior firm benefit from increasing its quality to the superior firm's level, but the superior firm is better off as well. Therefore, helping the inferior firm improve is a win-win strategy. In a complementary paper, Sajeesh and Raju (2010) examine the impact of variety seeking on location competition in a two-period horizontal Hotelling model. They show that if a subset of consumers have a preference for variety, then equilibrium levels of product differentiation will be lower than the level that would be obtained without a consumer preference for variety, though not to the minimum differentiation we find. In contrast with our results, Sajeesh and Raju's (2010) model finds the presence of variety-seeking consumers always reduces profits. Guo (2006) presents another related model in a horizontally differentiated setting by examining a duopoly with forwardbuying consumers who are uncertain of their future preferences. Such consumers sometimes make multiple purchases-mimicking the behavior of variety seeking - to remain flexible at the time of consumption. Guo (2006) shows that this behavior can also soften price competition. 
In some of the industries we consider, sellers offer multiunit discounts. For example, large ski resorts sell two-day tickets for less than twice the price of a oneday ticket. In our third main finding, we show that our first finding is robust to the presence of multiunit discounts: Stronger preferences for variety continue to soften price competition when qualities are similar and intensify price competition when qualities are dissimilar. In both settings, at least one firm uses a discount, and the effect of discounting on profits again depends on the extent of vertical differentiation and the strength of preference for variety.

\section{Model}

We model the competition between two firms as a two-stage game. In the first stage, the firms simultaneously select quality $q$ from an interval of feasible qualities. In the second stage, the firms set prices simultaneously, and consumer demand is realized. We now outline the notation of our model before discussing our assumptions in detail.

Firms: Two firms exist with identities $j=1$, 2. Each firm provides a distinct experience and sets its own quality level. In the first stage, firm $j$ selects quality $q_{j} \in[q, \bar{q}]$, where $q \geq 0$. The fixed cost $C(q)$ of selecting a given quality level is weakly increasing and convex in $q$ : $C^{\prime}(q) \geq 0, C^{\prime \prime}(q) \geq 0$. In the second stage, each firm $j$ charges a price $p_{j}$ for each unit of its good. We assume the marginal costs of production are constant across the two firms, so they can be normalized to zero without loss of generality. When the two firms select different qualities $L \equiv q_{j}<q_{-j} \equiv H$ in the first stage, we label the firms $L$ (for "low") and $H$ (for "high") according to the relative magnitude of their qualities. This conflation of identity and quality into a single index will clarify our exposition of the secondstage pricing game.

Consumers: Consumers have utility for up to two units of the good, and they differ in their marginal utility for quality $\theta$. Consumer type $\theta$ is distributed across the population uniformly on the $[0,1]$ interval. We normalize the utility of consuming no units to be zero. When consumer $\theta$ buys only one unit of the good and chooses firm $j$, his utility is standard $U_{j}^{1}=\beta+\theta q_{j}-p_{j}$. When consumer $\theta$ buys two units of the good, he cares about the identities of both products because of his preference for a variety of experiences. If the consumer buys one unit from each firm, he obtains a utility of $U_{V}=2 \beta+\theta \sum q_{j}-\sum p_{j}$ (when the two firms select different qualities $L \equiv q_{j}<q_{-j} \equiv H$ in the first stage, the utility is $U_{V}=2 \beta+\theta(L+H)-$ $\left.\left(p_{L}+p_{H}\right)\right)$. The indirect utility of consumer $\theta$ who buys two units from firm $j$ is $U_{j}=2 \beta+(1+\delta) \theta q_{j}-$ $2 p_{j}$, where $\delta<1$ reflects the rate at which perceived quality is diminishing across units of the same firm's product. As is common in these types of models, we assume $\beta$ is large enough that all consumers buy two units of the good in equilibrium. Furthermore, because $\beta$ appears additively in all two-unit utilities, we drop it from any utility calculations in the rest of the paper.

Two quality ranges: As noted in the introduction, we show that consumer behavior and the intensity of price competition depend crucially on the relative difference between the two firms' qualities and the degree of diminishing marginal utility $\delta$. We analyze the following two regions of the $\left(q_{1}, q_{2}, \delta\right)$ parameter space for which the price competition equilibrium is tractable to us:

Definition 1. For any fixed $\delta$, two firm qualities $0<L \leq H$ are similar if $L \geq((1+4 \delta) /(4+\delta)) H$, and dissimilar when $L \leq((\delta-\alpha) /(1-\alpha \delta)) H$, where $\alpha \equiv$ $(8 \sqrt{10}-17) / 39$.

From a consumer's perspective, similar qualities imply $L>\delta H$, so the variety bundle is the highest utility consumption alternative: all consumers would choose to purchase one unit from each firm if the two firms charged the same price. In contrast, dissimilar qualities imply $L<\delta H$, and two $H$ units are the highest utility consumption alternative. Note the definition intertwines the difference in qualities with the strength of preference for variety. For example, an alternative interpretation of similar qualities is that the preference for variety needs to be strong enough for a given fixed pair of qualities.

Having outlined the model, we now discuss its key assumptions. The main departure from standard models of vertical differentiation (Shaked and Sutton 1982, Moorthy 1988) is the diminishing marginal utility for multiple units of the same firm's product $(\delta<1)$ that captures a preference for variety described in the introduction. We further assume the preference for variety is proportional to the preference for quality $(\theta)$, consistent with the notion that connoisseurs experience a greater taste for variety compared to casual users. That is, we expect ski enthusiasts to value skiing at different resorts more than casual skiers, and for foodies to place the highest value on trying new restaurants. ${ }^{2}$

The main departure from previous models of competition with a consumer preference for variety is our focus on vertically differentiated firms and their inherent asymmetry. Our model thus applies in markets in which consumers care more about quality and novelty than about other attributes. In particular, consumers care more about having a variety of experiences than

\footnotetext{
${ }^{2}$ Many of our results continue to hold when we consider a constant additive disutility from repeated purchases instead. In particular, firms would still earn positive profits if they both offered identical qualities, and the profits increase with a stronger preference for variety. Please contact the authors for details.
} 
about which particular experience they have. For example, two ski resorts may have different scenic views in addition to different overall qualities, but the skiers care much more about quality and seeing a variety of views than about looking at one view versus the other. This conceptualization of variety seeking is consistent with the conceptualization of the need for trying new experiences to combat boredom or to satisfy curiosity; see Faison (1977) or Raju (1980).

Another departure from previous models of competition with a consumer preference for variety (Seetharaman and Che 2009, Sajeesh and Raju 2010) is that we abstract from dynamic considerations and examine a static model in which the price of the first unit is the same as the price of the second unit. This assumption acknowledges menu costs and the fact that in the industries we consider-ski resorts, amusement parks, and restaurants-prices do not tend to vary on a consumption-experience basis within the time frames we consider, such as a weekend. One can also think of our consumers as planning and purchasing both units of the good ahead of the actual experiences, as in Guo (2006).

\subsection{Consumer Behavior}

Suppose the two firms offer different qualities $L \equiv$ $q_{j}<q_{-j} \equiv H$. We assume market coverage, so consumers have three purchase choices: 2 units of $H$, 2 units of $L$, or 1 of each, which we call "variety." We denote the utilities from these choices as $U_{H}, U_{L}$ and $U_{V}$, respectively. The following inequalities determine the optimal decision of a consumer $\theta$ :

$$
\begin{aligned}
U_{V}>U_{L} & \Leftrightarrow(L+H) \theta-p_{H}-p_{L}>(1+\delta) L \theta-2 p_{L} \\
& \Leftrightarrow \theta>\frac{p_{H}-p_{L}}{H-\delta L}
\end{aligned}
$$

$$
\begin{aligned}
U_{H}>U_{L} & \Leftrightarrow(1+\delta)(H-L) \theta>2\left(p_{H}-p_{L}\right) \\
& \Leftrightarrow \theta>\frac{2\left(p_{H}-p_{L}\right)}{(1+\delta)(H-L)} .
\end{aligned}
$$

The $U_{V}$ versus $U_{H}$ comparison depends on whether $L>\delta H$ or $L<\delta H$. We discuss the two cases in turn. When $L$ and $H$ are similar (Definition 1) then $L>\delta H$, and the utility comparison becomes

$$
\begin{aligned}
U_{V}>U_{H} & \Leftrightarrow(L+H) \theta-p_{H}-p_{L}>(1+\delta) H \theta-2 p_{H} \\
& \Leftrightarrow \theta>\frac{p_{L}-p_{H}}{L-\delta H} .
\end{aligned}
$$

Figure 1 provides an illustration of consumer behavior. When $L$ and $H$ are similar, high- $\theta$ consumers choose variety, whereas the rest of the consumers buy two units of the cheaper good. To understand this pattern, note that when the superior good is cheaper $\left(p_{H}<p_{L}\right)$, no one buys two low-quality units, and consumers choose between variety and two high-quality units. On the other hand, when the superior good is more expensive $\left(p_{H}>p_{L}\right)$, no one buys two units of it: Whoever prefers $\{H, \delta H\}$ to $\{L, \delta L\}$ will also prefer the variety bundle $\{L, H\}$ to $\{H, \delta H\}$ because it gives more quality $(L>\delta H)$ for less money $\left(p_{H}+p_{L}<\right.$ $\left.2 p_{H}\right)$. Therefore, $p_{H}>p_{L}$ makes the consumers choose between variety and buying two low-quality units.

Note that equal prices $\left(p_{H}=p_{L}\right)$ make all consumers choose variety under similar qualities, so consumer behavior transitions continuously from one ordering of prices to the other. In other words, the demand functions of both firms are continuous at $p_{H}=p_{L}$, but the slopes of the demand functions change at that point. Also note that if the differences in prices are high enough, consumers will only purchase the cheaper product, and the more expensive product can be foreclosed out of the market.

\section{Figure 1 Consumer Behavior}
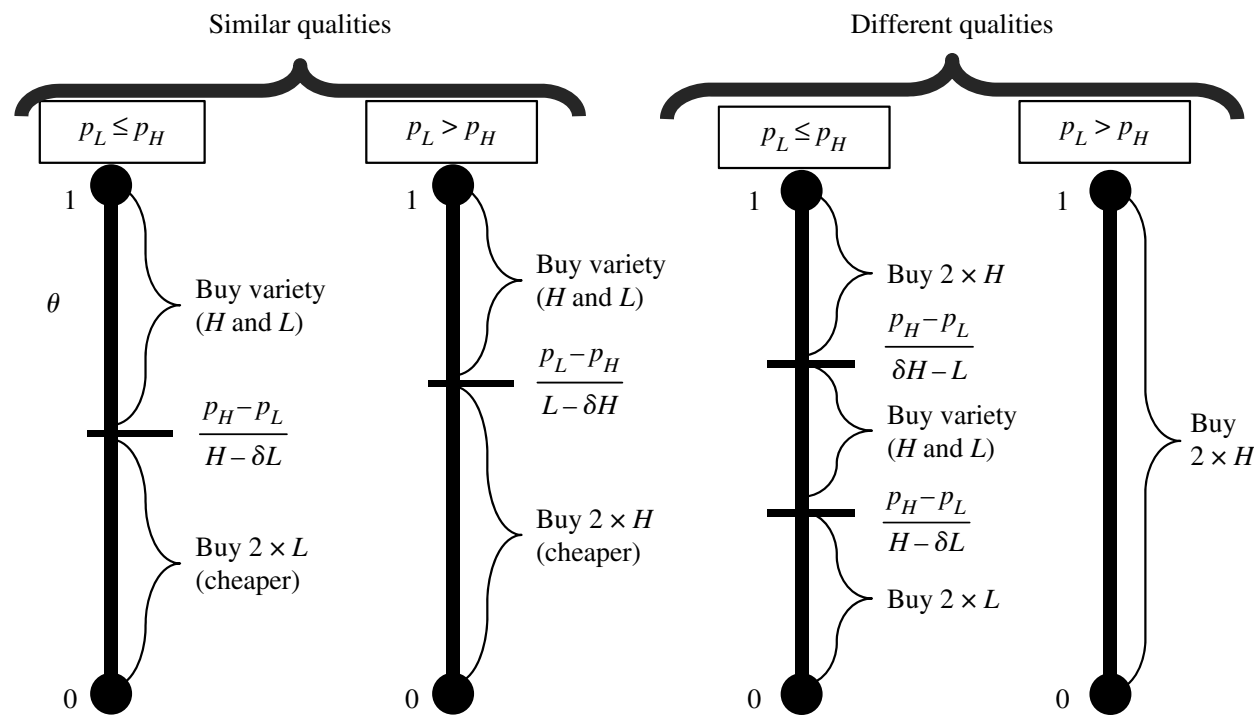
Now consider the $U_{V}$ versus $U_{H}$ comparison when $L$ and $H$ are dissimilar:

$$
\begin{aligned}
U_{V}>U_{H} & \Leftrightarrow(L+H) \theta-p_{H}-p_{L}>(1+\delta) H \theta-2 p_{H} \\
& \Leftrightarrow \theta<\frac{p_{H}-p_{L}}{\delta H-L} .
\end{aligned}
$$

Figure 1 (right side) illustrates consumer behavior under dissimilar qualities. Comparing Equation (3S) with Equation (3D), we see that the variety bundle $\{L, H\}$ is the highest-quality consumption alternative in similar-quality scenarios, so variety-seeking behavior occurs "at the top"; that is, the consumers with high value on quality $(\theta)$ choose variety. In dissimilar quality settings, the variety bundle $\{L, H\}$ is inferior to buying two units of $H$ (because $L<\delta H$ ), so the consumers with high $\theta$ buy two $H$ units. Varietyseeking behavior instead emerges "in the middle" of the $\theta$ spectrum because the variety bundle becomes a middle-quality option with a mid-level price. ${ }^{3}$ Therefore, unlike in the case of similar qualities, dissimilar qualities allow all three consumption choices to occur at a given set of prices.

To complete the description of consumer behavior, we note that the similar-qualities case with $L=H=q$ captures the behavior when both firms offer the same quality $q$. In such a case, high- $\theta$ consumers still choose variety, whereas low- $\theta$ consumers buy two units of the cheaper good; if both firms charge equal prices, all consumers purchase one unit from each firm.

\subsection{Price Competition}

We first show that when the two firms have similar qualities (per Definition 1), both firms profit more than they would in the absence of a consumer preference for variety $(\delta=1)$, and profits for both firms increase with the consumer preference for variety (i.e., as $\delta$ decreases). We then show that the opposite result occurs under dissimilar qualities: Both firms profit less than they would without a consumer preference for variety, and profits of both firms decrease as $\delta$ decreases.

\subsubsection{Price Competition with Similar Qualities.} When the two firms have similar but not equal qualities $(L<H)$, no pure-strategy pricing equilibrium exists because the high-quality firm's demand curve is kinked the wrong way at $p_{H}=p_{L}$ : The slope of its demand curve is steeper when $p_{H}>p_{L}$ than when $p_{H}<p_{L}$. The kink results from a switch in the type of indifferent customer at $p_{H}=p_{L}$ : When $p_{H}>p_{L}$, the indifferent consumer is deciding between variety and

\footnotetext{
${ }^{3}$ This assumes that the inferior good is cheaper, as it will be in equilibrium. When $p_{H}<p_{L}$, all (1), (2), and (3D) thresholds are negative, so all consumers consume two units of the high-quality good. This cannot occur in equilibrium because the low-quality firm can profitably deviate by lowering its price.
}

two units of the (cheaper) low-quality good. However, when $p_{H}<p_{L}$, the indifferent consumer is deciding between variety and two units of the (cheaper) high-quality good. One result of this kink is that the high firm wants to undercut high $p_{L}$ and overshoot low $p_{L}$, destroying the possibility of a purestrategy equilibrium. More details are available from the authors. Although no pure-strategy equilibrium exists, the game does have a tractable mixed-strategy equilibrium:

Proposition 1 (Price Competition with Similar Qualities). When the firms have similar qualities, an equilibrium exists in which the low-quality firm sets a price of $p_{L}=\sqrt{(H-\delta L)(L-\delta H)}$ and the high-quality firm plays a mixed strategy, pricing at $p_{H}^{\text {up }}=(H-\delta L+$ $\sqrt{(H-\delta L)(L-\delta H)}) / 2$ with probability $\operatorname{Pr}\left(p_{H}^{\text {up }}\right)=$ $\sqrt{(H-\delta L) /((H+L)(1-\delta))}$, and $p_{H}^{\text {down }}=(L-\delta H+$ $\sqrt{(H-\delta L)(L-\delta H)}) / 2$ with probability $1-\operatorname{Pr}\left(p_{H}^{\text {up }}\right)$. Firm profits are $\Pi_{H}=((\sqrt{H-\delta L}+\sqrt{L-\delta H}) / 2)^{2}>\Pi_{L}=$ $\sqrt{(H-\delta L)(L-\delta H)}$. Profits of both firms increase as $\delta$ decreases (i.e., as preference for variety increases).

The proofs of all propositions appear in the appendix. The intuition behind Proposition 1 is that $p_{L}=$ $\sqrt{(H-\delta L)(L-\delta H)}$, which is between $L-\delta H$ and $H-\delta L$, makes the high-quality firm indifferent between undercutting $p_{L}$ and overshooting $p_{L}$. The low-quality firm's demand in the face of a highquality firm mixing between two points is concave, and $\operatorname{Pr}\left(p_{H}^{\text {up }}\right)$ is set such that $\sqrt{(H-\delta L)(L-\delta H)}$ is the low-quality firm's best response to the high firm's mixed strategy.

Proposition 1 also covers competition between two firms that offer equal qualities $(L=H=q)$. In such a case, $p_{H}^{\text {up }}=p_{H}^{\text {down }}$ and the game has a pure-strategy equilibrium, which is possible because equalizing the firm qualities removes the kinks in the demand functions. Profits and prices are continuous as $L$ approaches $H$ because the pure-strategy equilibrium is the limit of the equilibrium in Proposition 1. The equal-quality case clearly demonstrates how consumer preferences soften price competition: In the absence of tastes for variety $(\delta=1)$, the firms earn zero profits when they offer identical products. When consumers have a taste for variety $(\delta<1)$, the firms earn positive profits and these profits increase as $\delta$ decreases. We summarize the equal-quality results in a corollary:

Corollary. When $L=H=q$, a pure-strategy equilibrium exists such that $p_{q}=\Pi_{q}=q(1-\delta)$.

The equilibrium of Proposition 1 has intuitive comparative statics: The high firm earns greater equilibrium profits than the low firm and is more likely to overshoot than undercut the low firm's pure strategy. Also, greater preferences for variety increase both 
firms' profits. Intuitively, the preference for variety softens competition by ensuring that a higher-priced firm gets positive demand if price differences are small enough. As we will see below, this intuition only holds when the firms offer similar qualities.

2.2.2. Price Competition with Dissimilar Qualities. When the two firm qualities are dissimilar in the sense of Definition 1, a pure-strategy pricing equilibrium exists with the low-quality firm charging less than the high-quality firm:

Proposition 2 (Price Competition with DissimILAR QuALITIES). When the firms select dissimilar qualities $L<H$, a unique pure-strategy Nash equilibrium exists with $p_{L}=(2 / 3) R$ and $p_{H}=(4 / 3) R$, where

$$
R=\frac{(\delta H-L)(H-\delta L)}{(1+\delta)(H-L)} \text {. }
$$

Firm profits are $\Pi_{L}=(4 / 9) R<\Pi_{H}=(16 / 9) R$. Profits of both firms decrease as $\delta$ decreases (i.e., as preference for variety increases).

The computations behind the equilibrium in Proposition 2 are standard, and the equilibrium has many of the standard properties of price-competition equilibria in vertically differentiated markets as originally described by Shaked and Sutton (1982): The highquality firm charges a higher price and earns higher profits than the low-quality firm, and profits for both firms increase as the amount of vertical differentiation increases. The parallel is more than qualitative. The profit functions presented in Proposition 2 reduce to (twice, for two units) the profit functions from Shaked and Sutton when $\delta=1$.

In contrast with the similar-qualities case, consumer preferences for variety intensify the price competition here. This contrast is a result of variety-seeking behavior shifting from the top to the middle of the $\theta$ spectrum: Because the variety bundle is a middle-quality option under dissimilar qualities, the firms have to compete for two sets of marginal consumers: High $\theta$ consumers decide between variety and two $H$ units, and low $\theta$ consumers decide between variety and two $L$ units. In other words, the firms compete intensely to sell the consumer the second unit, and each firm's second unit effectively competes with the competitor's first unit. The crucial match-up is between the second superior unit that delivers $\delta H$ perceived quality and the first inferior unit that delivers $L$. Note that the smaller the $\delta$ (i.e., the stronger preference for variety is), the less differentiated these two units are, and so price competition intensifies.

\subsection{Equilibrium Quality Choice}

Having analyzed the price competition in the second stage, we now solve the first stage of the game where firms choose qualities. We focus most on the case where qualities are constrained to be similar. ${ }^{4}$ We then briefly discuss what happens under dissimilar qualities.

Proposition 3 (Quality Competition EquilibRIUM WITH Similar Qualities). Suppose the range of feasible qualities $q_{j} \in[q, \bar{q}]$ is restricted to similar qualities; that is, $q \geq((1+\overline{4} \delta) /(4+\delta)) \bar{q}$. Then a unique purestrategy first-stage equilibrium exists in which both firms select the same quality $\min (\bar{q}, \tilde{q})$, where $\tilde{q}$ is either the lower bound $q$ when $C^{\prime}(q)>(1-\delta) / 2$ or the quality level above q, where $C^{\prime}(\tilde{q})=(\overline{1}-\delta) / 2$.

A crucial component of the forces behind Proposition 3 is that when $L<H$ and $C^{\prime}(H)<(1-\delta) / 2$, the low-quality firm wants to increase its quality to at least that of the high-quality firm. Similarly, each firm wants to raise its quality level if both firms have equal qualities $q=q_{1}=q_{2}$, as long as $C^{\prime}(q)<(1-\delta) / 2$. Therefore, the qualities of both firms rise as they best respond to each other, until they reach the point at which $C^{\prime}(\tilde{q})=(1-\delta) / 2$, unless this value of $\tilde{q}$ falls outside the feasible range. In equilibrium, the firms will always choose the same quality, leading to minimum differentiation.

Proposition 3 contrasts with the basic result in vertically differentiated markets without a taste for variety, where Shaked and Sutton (1982) and Moorthy (1988) show that firms want to differentiate themselves to soften price competition. Our result is in the same direction as the findings of Sajeesh and Raju (2010), who find that preferences for variety reduce the equilibrium level of product differentiation in a horizontal-differentiation model, although they do not find minimum differentiation as we do. Under Sajeesh and Raju's model, margins for each firm shrink to zero under minimum differentiation. So the underlying mechanics driving the results are different in the two settings, and variety seeking appears to soften location competition more in vertical settings than in horizontal settings.

The intuition behind Proposition 3 is that preferences for variety soften price competition the most when the firms have the same qualities. Each firm easily sells one unit to every consumer, but a unilateral price reduction does not convince many consumers to purchase a second unit. As the quality of the inferior firm decreases, the superior firm has more success selling some consumers a second unit, and so

\footnotetext{
${ }^{4}$ Restricting the range of feasible qualities is often realistic because of technology or legal constraints. For example, health standards set a minimum allowable quality level for restaurants. In resort industries, natural conditions such as snowfall or sunshine fix a large component of the quality, often restricting nearby resorts to offer similar qualities.
} 
price competition intensifies. Comparing our results with the monopoly benchmark with zero marginal costs further clarifies the intuition by showing that minimum differentiation arises from equilibrium considerations rather than from the structure of demand. In the appendix, we show that the monopolist selects maximally different qualities to price discriminate. In contrast, two competing firms benefit more from creating increased consumer value. Therefore, the equilibrium of Proposition 3 is a result of active price competition; the two firms do not somehow implement the monopoly strategy and split the profits.

Proposition 3 appears to be consistent with an intuition that the preferences for variety create an additional dimension of differentiation for the second units, and it is the additional differentiation that allows both firms to make profits with equal qualities. This intuition is valid in broad terms, but the relationship between vertical differentiation and the strength of variety seeking is more subtle than a relationship between two generic dimensions of differentiation. Specifically, the preferences for variety only mimic additional differentiation when the two products have similar qualities. When the products have different qualities, the preferences for variety reduce the effective vertical differentiation because stronger preferences for variety make the second superior unit more similar to the first inferior unit it competes with in the market. Thus, the extent to which preferences for variety mimic differentiation along another dimension depends on the level of differentiation between the firms.

Proposition 3 relies on the low-quality firm making more money as it increases its quality up to the highquality firm's level. The reduction in price competition from bringing the firm qualities closer together is so strong that even the higher-quality firm benefits as $L$ approaches $H$ :

Proposition 4 (Improving the Weaker CompetiTOR Is WIN-WIN). When two firms have similar, but not equal, qualities, the higher-quality firm benefits from an increase in the lower-quality firm's quality.

The proof of Proposition 4 is simple:

$$
\frac{\partial \Pi_{H}}{\partial L}=\frac{\sqrt{H-\delta L}-\delta \sqrt{L-\delta H}}{4 \sqrt{(H-\delta L)(L-\delta H)}}(\sqrt{H-\delta L}+\sqrt{L-\delta H})>0 .
$$

Thus, both firms are better off under minimum differentiation. In other words, both firms providing an equal level of quality is not just an equilibrium outcome, but also a win-win scenario for both firms.

We summarize the profits from Propositions 1, 3, and 4 with $C=0$ and $H=\bar{q}$ (so both firms choose quality $\bar{q}$ in equilibrium) in Figure 2. Both firms earn the greatest profits under minimum differentiation,
Figure 2 Equilibrium Profits Under Similar Qualities

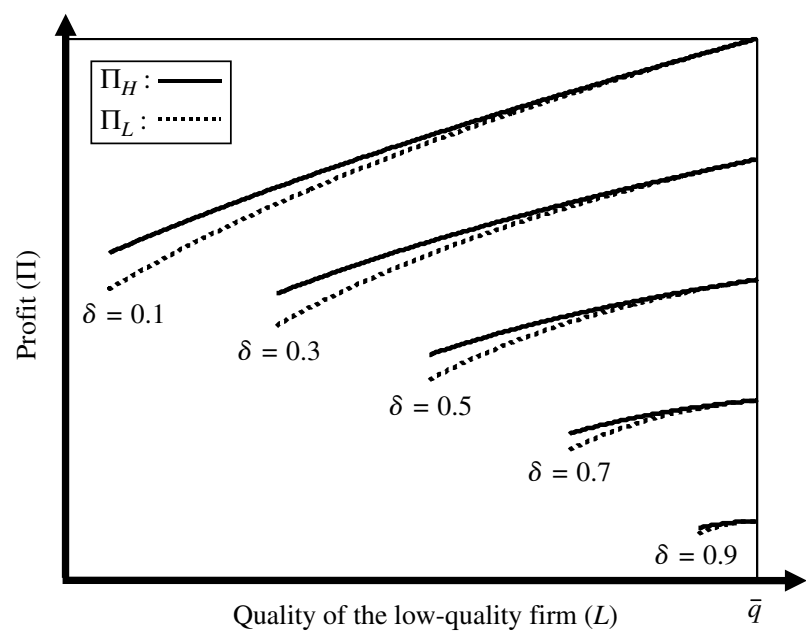

Notes. Each line shows the relationship between the low firm's quality (conditional on $H=\bar{q}$ ) and profits for different $\delta$. Each line starts at the lowest $L$ possible under Definition 1 of similar qualities.

and the profits decrease as the preference for variety decreases (i.e., as $\delta$ increases).

We now briefly consider the case where the two firms have dissimilar qualities. In such a situation, profits increase for both firms with greater levels of differentiation (at least in a local sense); that is, profits for both firms are higher as the quality of $H$ is higher or the quality of $L$ is lower. The reasons for the contrast between the results under similar qualities and the results under differentiated qualities are that if the products start out differentiated enough that middle- $\theta$ consumers choose variety, then differentiating further increases the amount of differentiation between the second unit of $H$ and the first unit of $L$, leading to softer competition. In contrast, under similar qualities, greater quality differences lead to lower differentiation between a second unit of $H$ and a first unit of $L$.

Note that we limit our analysis to two restricted sets of feasible qualities from Definition 1 because an intermediate (greater than similar, but smaller than dissimilar) range of relative qualities $L / H \in$ $[(\delta-\alpha) /(1-\alpha \delta),(1+4 \delta) /(4+\delta)]$ exists with no purestrategy pricing equilibria, and no mixed-strategy equilibria are tractable to us. We hope that future work can address this gap in our analysis, and we conjecture that price competition is so intense in the intermediate region that endogenous equilibrium qualities never end up there. Based on this conjecture, it makes sense to ask whether profits are higher under minimum versus maximum vertical differentiation. Comparing the profits from minimum versus maximum differentiation, we find that the low-quality firm prefers minimum differentiation to maximum differentiation when $\delta$ is low enough (customers have a strong enough preference for variety seeking). 


\section{Extension: Volume Discounts}

In some of the industries we consider, some sellers offer multiunit discounts. For example, large ski resorts often sell two-day tickets for less than twice the price of a one-day ticket. We examine the impact of volume discounts on the intensity of price competition for two quality configurations: $L=H=q$ (identical qualities) and $L<\delta H$ (dissimilar qualities). Our first main finding is robust to volume discounts: Stronger preferences for variety continue to soften competition when qualities are identical and to intensify price competition when qualities are dissimilar. We also compare the profits under volume discounting to profits under unit pricing, and we find another dichotomy: Volume discounting intensifies price competition when qualities are identical, but it softens it when qualities are dissimilar.

Suppose the firms can discount the second unit, and let the discounted price of two units from firm $j$ be $\left(1+\lambda_{j}\right) p_{j}$ for some $0 \leq \lambda_{j} \leq 1$. The utility comparisons of Equations (1) and (2) change to

$$
\begin{aligned}
U_{V}>U_{L} & \Leftrightarrow(L+H) \theta-p_{H}-p_{L}>(1+\delta) L \theta-\left(1+\lambda_{L}\right) p_{L} \\
& \Leftrightarrow \theta>\frac{p_{H}-\lambda_{L} p_{L}}{H-\delta L} \equiv \theta_{L}, \\
U_{H}>U_{L} & \Leftrightarrow(1+\delta)(H-L) \theta>\left(1+\lambda_{H}\right) p_{H}-\left(1+\lambda_{L}\right) p_{L} \\
& \Leftrightarrow \theta>\frac{p_{H}+\lambda_{H} p_{H}-p_{L}-\lambda_{L} p_{L}}{(1+\delta)(H-L)} \equiv \theta_{2} .
\end{aligned}
$$

Consider first what happens in the similar-qualities case. Equation (3S) changes to

$$
\begin{aligned}
U_{V}>U_{H} & \Leftrightarrow(L+H) \theta-p_{H}-p_{L} \\
& >(1+\delta) H \theta-\left(1+\lambda_{H}\right) p_{H} \\
& \Leftrightarrow \theta>\frac{p_{L}-\lambda_{H} p_{H}}{L-\delta H} \equiv \theta_{H} .
\end{aligned}
$$

The consumer behavior is analogous to the similarqualities unit-pricing situation illustrated in Figure 1, with high- $\theta$ customers choosing variety. No purestrategy equilibria exist under similar qualities, and no tractable mixed-strategy equilibria exist when $L$ is slightly less than $H$. In the identical-quality case (relevant because it is the equilibrium configuration under unit pricing; see Proposition 3), we find a tractable symmetric mixed-strategy equilibrium:

Proposition 5. When the firms offer identical qualities $(L=H=q)$ and each firm can offer a volume discount $0 \leq \lambda_{j} \leq 1$, a symmetric mixed-strategy Nash equilibrium exists in which both firms include the second unit for free with the first unit $\left(\lambda_{j}=0\right)$. Each firm draws its two-unit price $p$ from the distribution with a cumulative distribution function of $F(p)=q(1-\delta)[4 p-q(1-\delta)] /\left(4 p^{2}\right)$ on the support $[q(1-\delta) / 4, q(1-\delta) / 2]$, and each firm's expected profits are $\Pi=q(1-\delta) / 4$, which is less than the equilibrium profit under unit pricing.
The proof proceeds by first solving the game between two firms restricted to including the second unit for free with the first unit. We then consider a deviation to any smaller second-unit discount by one of the firms, and show that such a deviation cannot be profitable. Intuitively, given a fixed effective two-unit price of $\left(1+\lambda_{i}\right) p_{i}$, the second-unit discount becomes a transfer to variety seekers who have a high willingness to pay, but only for the first unit from each firm. Thus, the best response to an opponent who offers the second unit for free with the first unit is to follow suit. Both firms thus resort to pure bundling in equilibrium despite having the freedom to use a fully nonlinear pricing schedule.

Comparing profits between Propositions 1 and 5, volume discounting clearly reduces profits (by a factor of four). However, as in Proposition 1, profits of both firms increase as $\delta$ decreases (i.e., as preference for variety increases). Therefore, the effect of the strength of preference for variety on price competition we found in Proposition 1 is robust to the presence of multiunit discounts in the case of equal qualities. Moreover, the fact that profits remain positive means the preference for variety continues to soften price competition even when the firms can use volume discounts: If consumers instead cared only about quality and the firms offered products with identical qualities, Bertrand price competition would wipe out all profits.

When qualities are exogenously set at dissimilar levels, the analysis simplifies because a pure-strategy equilibrium exists. From a consumer-behavior perspective, the only change from $\S 2$ is the cutoff between buying variety and buying two $H$ units:

$$
U_{V}>U_{H} \Leftrightarrow \theta<\frac{\lambda_{H} p_{H}-p_{L}}{\delta H-L} \equiv \theta_{H}
$$

As in the proof of Proposition 5, we show firms that can set any multiunit discounts obtain the same equilibrium profits as they would if they were restricted to include the second unit for free with the first unit (even though only the high-quality firm actually discounts in equilibrium). Unlike in the equal-quality case, discounting softens competition here:

Proposition 6. When the two firms offer dissimilar qualities $L<H$ and each firm can set a volume discount $0 \leq \lambda_{j} \leq 1$, an equilibrium exists in which $L$ charges $p_{L}=$ $(H-L)(1+\delta) / 6$ for each unit $\left(\lambda_{L}=1\right)$ and $H$ offers the second unit for free with the first unit $\left(\lambda_{H}=0\right)$, for which it charges $p_{H}=2(H-L)(1+\delta) / 3$. The equilibrium profits are $\Pi_{H}=4(H-L)(1+\delta) / 9>(H-L)(1+\delta) / 9=\Pi_{L}$. Each firm's profit exceeds its profit under unit pricing.

No consumers choose variety in the equilibrium because $\lambda_{H}=0$ and any customer who has bought two high-quality units will never buy any low-quality 
products ( $L<\delta H$, so buying even one unit of $L$ would involve an expense without any incremental benefit). Therefore, the competition over two-unit bundles $\left(\lambda_{j}=0\right)$ is akin to a standard unit-demand duopoly with the difference in product qualities being the difference between the utilities of the two bundles, $(H-L)(1+\delta)$. When the firms can use any volume discounts, some consumers choose variety if $\lambda_{H}>0$, but we show that the high-quality firm always wants to engage in pure bundling to get rid of varietyseeking behavior. The low firm, on the other hand, weakly prefers unit pricing: When the high firm plays its equilibrium strategy, only the total price of the low-quality bundle matters, but $\lambda_{H}>0$ makes the fullprice competition $p_{H}$ for the second $L$ unit less intense than the discounted $\lambda_{H} p_{H}$ competition for the first $L$ unit. Because $\lambda_{H}=0$ in equilibrium, both firms make the same profits they would earn if they were both restricted to offering pure bundles $\left(\lambda_{j}=0\right)$.

Unlike in Proposition 5, allowing the firms to use multiunit discounts softens competition relative to simple unit pricing. Intuitively, discounting is less competitive with dissimilar qualities because no consumers choose variety in equilibrium and the price competition evolves as if the sellers were selling vertically differentiated products with a single marginal customer in the middle. Although the level of profit changes compared to those in Proposition 2, profits of both firms decrease as $\delta$ decreases (i.e., as preference for variety increases). Therefore, the direction the preference for variety has on price competition in Proposition 2 is robust to the presence of multiunit discounts.

\section{Discussion}

In a vertically differentiated duopoly, consumer preference for variety can have substantial effects on three managerially important outcomes: price competition, quality competition, and the profitability of discounting. We find that the direction of all three effects depends closely on whether two units of the superiorfirm product deliver more or less utility than a variety bundle (i.e., one unit from each firm).

When the firm qualities are similar enough and/or the preference for variety is strong enough that the variety bundle provides the highest consumption utility, consumer preferences for variety soften price competition (and increase profits). The reduction in price competition is so strong that upfront quality competition results in both firms choosing identical qualities. The quality competition result runs counter to the principle of vertical differentiation established by Shaked and Sutton (1982) and Moorthy (1988). The intuition for our result is that when firms produce products of the same quality, they find it difficult to sell many customers a second unit of the good without steeply decreasing their prices, so they choose not to target that market. We therefore predict co-located competitors in experience goods with strong customer preferences for variety, such as restaurants or resorts, are more likely to offer similar qualities than those in markets in which customers do not have preferences for variety. For example, imagine two restaurants in an isolated city where tourists often spend a weekend. Variety seeking is strong in the restaurant industry in that tourists would rather visit two restaurants than the same restaurant twice, so we would expect the restaurants to offer identical qualities. Consider instead the market for motel rooms in the same city. Consumers do not value variety as much in that industry, so we would expect firms to offer different qualities, as in Mazzeo (2002).

Note the result that firms want minimum differentiation (if the scope of differentiation is limited enough) is not propagated purely by firms choosing to increase their qualities; the high-quality firm is better off if the lower-quality firm chooses a higher quality rather than a lower quality. In other words, the superior firm would be willing to expend resources to improve its rival's products. In the restaurant example, suppose one of the two chefs is excellent and the other is also very good, but not as great. Our model demonstrates that the excellent chef has an incentive to help the weaker chef become a better cook, as long as the weaker chef has a similar-enough initial quality. In the resulting equilibrium, the restaurants offer the same quality, charge the same price, and all consumers choose variety.

When qualities are not only similar but actually identical, the consumer preference for variety gives both firms an incentive to use "buy-one-get-one-free" discounts. Allowing both firms to use volume discounts reduces profits relative to unit pricing, but stronger preferences for variety continue to soften competition.

All three effects of consumer preferences for variety are reversed when the firm qualities are dissimilar enough and/or the preference for variety is weak enough that two high-quality units provide more consumption utility than the variety bundle. Then, consumer preferences for variety intensify price competition (and reduce profits) whether or not firms can use volume discounts. Counter intuitively, our model predicts that seemingly very differentiated competitors, such as a fancy restaurant and a pizza pub, in a setting that involves multiple purchase opportunities (e.g., a small, isolated resort town), may be competing more on price than they would if they were both located in an area that served only nonrepeat customers (e.g., an interstate highway stop). Also, we find that allowing both firms to use volume 
discounts increases profits relative to unit pricing, and that the lower-quality firm does not offer any discount in equilibrium. One may hypothesize that giving each firm more degrees of pricing freedom would intensify price competition (as it does under equal qualities). But under dissimilar qualities, volume discounts increase profits because they combat the profit destroying variety-seeking behavior. Finally, as to quality choice, when firms are differentiated enough, profits for both firms locally increase with greater amounts of differentiation.

\section{Acknowledgments}

The authors thank Xavier Drèze, Sridhar Moorthy, Peter Rossi, Miguel Villas-Boas, the associate editor, and two anonymous referees, as well as participants at the 2010 Invitational Choice Symposium, the 2011 University of California, Los Angeles Marketing Camp, and the 2010 Marketing Science Conference for their detailed and helpful comments. Both authors contributed equally to this paper.

\section{Appendix. Proofs of the Propositions}

Proof of Proposition 1. The equilibrium can be exposed most clearly in terms of the following quantities: $D \equiv H-\delta L, C \equiv L-\delta H, B \equiv \sqrt{L-\delta H}=\sqrt{C}, A \equiv \sqrt{H-\delta L}=$ $\sqrt{D}$. Under this notation, Assumption 1 is equivalent to $A<2 B \Leftrightarrow D<4 C$. Let $p_{L}=A B$, and note that $C<p_{L}<D$ because $A B$ is the geometric mean of $C$ and $D$. We first show the high firm is indifferent between undercutting and overshooting this $p_{L}$ : The optimal undercutting deviation is $p_{H}^{\text {down }}=(B(A+B)) / 2$ and yields a profit of $\Pi_{H}^{\text {down }}\left(p_{H}^{\text {down }}\right)=$ $\left(C+p_{L}\right)^{2} /(4 C)$. The undercut to $p_{H}^{\text {down }}$ does not drive the low firm out of the market because $\operatorname{Demand}_{L}\left(p_{L} \mid p_{H}=p_{H}^{\text {down }}\right)=$ $1-\left(p_{L}-p_{H}^{\text {down }}\right) / C>0 \Leftrightarrow A B-B(A+B) / 2<B^{2} \Leftrightarrow A<3 B$, which is in turn implied by Assumption 1. The optimal overshooting deviation is $p_{H}^{\text {up }}=A(A+B) / 2$, which yields a profit $\Pi_{H}^{\text {up }}=\left(D+p_{L}\right)^{2} /(4 D)$. Comparing the two profits, we find that $\Pi_{H}^{\text {up }}=\Pi_{H}^{\text {down }} \Leftrightarrow C\left(D^{2}+2 D p_{L}+p_{L}^{2}\right)=D\left(C^{2}+\right.$ $\left.2 C p_{L}+p_{L}^{2}\right) \Leftrightarrow D C=p_{L}^{2} \Leftrightarrow p_{L}=A B$. Because the high firm is indifferent between undercutting and overshooting $p_{L}=A B$, any mixed strategy that plays $p_{H}^{\text {up }}$ with probability $\rho$ and $p_{H}^{\text {down }}$ with probability $1-\rho$ is a high firm's best response to $p_{L}=A B$.

To close the equilibrium construction, we find $\rho$ such that $p_{L}=A B$ is the best response of the low firm. When the high firm plays a mixed strategy with a support $\left\{p_{H}^{\text {down }}, p_{H}^{\text {up }}\right\}$, the profit of the low firm is obviously increasing for small prices $\left(p_{L}<p_{H}^{\text {down }}\right)$, decreasing for large prices $\left(p_{L}>p_{H}^{\text {up }}\right)$, and continuous at $p_{L}=p_{H}^{\text {up }}$ and $p_{L}=p_{H}^{\text {down }}$. Therefore, the maximum must be somewhere in the $\left[p_{H}^{\text {down }}, p_{H}^{\text {up }}\right]$ interval, where the function is $\Pi_{L}=\rho p_{L}\left(1+\left(p_{H}^{\text {up }}-p_{L}\right) / D\right)+p_{L}(1-\rho) \mathbf{1}\left(p_{L}<\right.$ $\left.\min \left(p_{H}^{\text {up }}, p_{H}^{\text {down }}+C\right)\right)\left(\left(p_{H}^{\text {down }}-p_{L}\right) / C\right)$.

Because $p_{H}^{\text {down }}+C<p_{L}<p_{H}^{\text {up }}$ imply an increasing $\Pi_{L}$, the maximum must be either at $p_{L}=p_{H}^{\text {up }}$ or somewhere in the $\left[p_{H}^{\text {down }}, p_{H}^{\text {down }}+C\right]$ interval. To find the best price in $\left[p_{H}^{\text {down }}, p_{H}^{\text {down }}+C\right]$, let $p_{H}^{\text {down }}=B(A+B) / 2, p_{H}^{\text {up }}=A(A+B) / 2$ and note that the profit function for $p_{H}^{\text {down }}+C<p_{L}<p_{H}^{\text {up }}$ simplifies to $\Pi_{L}=p_{L}[1+((A+B) / 2)(\rho / A+(1-\rho) / B)-$ $\left.p_{L}\left(\rho / A^{2}+(1-\rho) / B^{2}\right)\right]$ which is concave. The first-order condition is $p_{L}=(A B / 4)\left(1+3 A B /\left((1-\rho) A^{2}+\rho B^{2}\right)\right)$. Solving for $\rho$ for $p_{L}=A B$ in turn yields $\rho^{*}=A /(A+B)$. Plugging $\rho^{*}$ back into the profit function yields $\Pi_{L}\left(A B \mid \rho^{*}\right)=A B$. When the high firm plays $\rho^{*}$, the first-order condition for the low firm has an interior solution inside the $\left[p_{H}^{\text {down }}, p_{H}^{\text {down }}+C\right]$ interval: $B(A+B) / 2<A B$ is obvious, and $A B<B(A+B) / 2+$ $B^{2} \Leftrightarrow A<3 B$, which is implied by similar qualities (Definition 1). The low firm prefers to play $p_{L}=A B$ to $p_{L}=p_{H}^{\text {up }}$. $\Pi_{L}\left(A B \mid \rho^{*}\right)=A B>\Pi_{L}\left(p_{H}^{\text {up }}\right)=\rho p_{H}^{\text {up }}=A^{2} / 2 \Leftrightarrow A<2 B$, which is exactly the definition of similar qualities. In equilibrium, the high firm makes $\Pi_{H}=((A+B) / 2)^{2}$, which exceeds the equilibrium profit of the low firm because the arithmetic mean always exceeds the geometric mean (Cauchy): $\Pi_{H}=$ $((A+B) / 2)^{2}>A B=\Pi_{L} \Leftrightarrow(A+B) / 2>\sqrt{A B}$. Finally, the $\partial \Pi_{H} / \partial \delta<0$ and $\partial \Pi_{L} / \partial \delta<0$ comparative statics are obvious. Now substitute $L=H=q$ in the profit functions and keeps track of firm identities: $\Pi_{1}\left(p_{1}\right)=p_{1}\left(1+\left(p_{2}-p_{1}\right) /(q(1-\delta))\right)$ and $\Pi_{2}^{\text {up }}\left(p_{2}\right)=\Pi_{2}^{\text {down }}\left(p_{2}\right)=p_{2}\left(1+\left(p_{1}-p_{2}\right) /(q(1-\delta))\right)$.

Neither firm's demand function has a kink now, and the profit functions are the same. Therefore, only $p_{1}=p_{2}$ can be a pure-strategy equilibrium. The profit functions are also concave, so the unique candidate prices $p_{1}=p_{2}=q(1-\delta)$ are indeed equilibrium prices. Q.E.D.

Proof of Proposition 2. The demand functions consumer behavior implies are

$$
\begin{gathered}
\text { Demand }_{L}=\frac{p_{H}-p_{L}}{H-\delta L}+\frac{p_{H}-p_{L}}{\delta H-L}=\frac{p_{H}-p_{L}}{R}, \\
\text { Demand }_{H}=2-\left(\frac{p_{H}-p_{L}}{H-\delta L}+\frac{p_{H}-p_{L}}{\delta H-L}\right)=\frac{2 R+p_{L}-p_{H}}{R},
\end{gathered}
$$

where $R=(\delta H-L)(H-\delta L) /((1+\delta)(H-L))$. Therefore, the profit functions are $\Pi_{L}\left(p_{L}\right)=p_{L}\left(p_{H}-p_{L}\right) / R$ and $\Pi_{H}\left(p_{H}\right)=$ $\left(p_{H} / R\right)\left[\left(p_{L}-p_{H}\right)+2 R\right]$. The local (within the ordering of the cutoffs) best-response functions are therefore $p_{L}=$ $p_{H} / 2, p_{H}=p_{L} / 2+R$, and candidate prices for equilibrium are $p_{L}=(2 / 3) R<p_{H}=(4 / 3) R$. It is easy to check that both marginal consumers lie within the support of $\theta: 0<2(\delta H-L) /(3(1+\delta)(H-L))=\left(p_{H}-p_{L}\right) /(H-\delta L)$ and $\left(p_{H}-p_{L}\right) /(\delta H-L)=2(H-\delta L) /(3(1+\delta)(H-L))<1$. The second constraint holds because $2(H-\delta L)<3(1+\delta)$. $(H-L) \Leftrightarrow(H-\delta L)+3(\delta H-L)>0$.

The profits in the candidate equilibrium are $\Pi_{L}=(4 / 9) R$, $\Pi_{H}\left(p_{H}\right)=(16 / 9) R$. The above situation is an equilibrium whenever the high firm does not want to deviate up to make money only on the variety seekers. The consumer behavior then becomes $\left[0,\left(p_{H}-p_{L}\right) /(H-\delta L)\right]: L$ both periods and $\left[\left(p_{H}-p_{L}\right) /(H-\delta L), 1\right]$ : variety seek. The best such deviation is $p_{H}=\left(H-\delta L+p_{L}\right) / 2=\left(3 D^{2}+5 C D\right) /(6(D+C))$, where $D=H-\delta L$ and $C=\delta H-L$. The best deviation yields a profit of $D((3 D+5 C) /(6(D+C)))^{2}$, so it is profitable when $D((3 D+5 C) /(6(D+C)))^{2}>16 C D /(9(D+C))$, which reduces to $9 D^{2}-34 C D-39 C^{2}>0$ and holds for $C=0$ and is decreasing for positive $C$ in $C$, so a cutoff $C^{*}<D$ exists, beyond which this deviation is not profitable. For all $C>C^{*}$, the above candidate equilibrium is an equilibrium. The cutoff is $C=((8 \sqrt{10}-17) / 39) D \approx 0.21 D$. To prove the comparative static in $\delta$, it is sufficient to show that $\partial R / \partial \delta>0$. $R$ increases in $\delta$ :

$$
\begin{aligned}
\frac{\partial R}{\partial \delta} & =\frac{H^{2}-\left(\delta^{2}+2 \delta-1\right) H L+L^{2}}{(1+\delta)^{2}(H-L)} \\
& =\frac{(H-L)^{2}+\left(3-2 \delta-\delta^{2}\right) H L}{(1+\delta)^{2}(H-L)}>0 . \quad \text { Q.E.D. }
\end{aligned}
$$


Proof of Proposition 3. Each firm is maximizing $\Pi_{q}-C(q)$. It is straightforward to confirm that $\Pi_{L}-C(L)$ and $\Pi_{H}-C(H)$ are both concave, so we can rely on first-order analysis. Differentiating $\Pi_{H}$ from Proposition 1 with respect to $H$ shows that each firm wants to deviate from $q=q_{1}=q_{2}<\bar{q}$ such that $C^{\prime}(q)<(1-\delta) / 2$ because $\partial \Pi_{H} /\left.\partial H\right|_{L=H=q}=(1-\delta) / 2$. Differentiating $\Pi_{L}$ from Proposition 1 with respect to $L$ shows that the lower-quality firm would always prefer to raise its quality up to any $H$ such that $C^{\prime}(H)<(1-\delta) / 2$ because $\partial \Pi_{L} / \partial L=((H-\delta L)-$ $\delta(L-\delta H)) /(2 \sqrt{(H-\delta L)(L-\delta H)})>(1-\delta) / 2 \Leftrightarrow \delta H<L$ and $C^{\prime \prime}>0$ means that $C^{\prime}(q)$ is increasing in $q$, and so $(\partial / \partial L)\left(\Pi_{L}-C(L)\right)>0$ for every $L<H$. Therefore, either $C^{\prime}(\bar{q})<(1-\delta) / 2$ and both firms run into the upper bound of the feasibility constraint or there is a $\tilde{q}<\bar{q}$ such that $C^{\prime}(\tilde{q})=$ $(1-\delta) / 2$, and neither firm wants to raise its quality further. Q.E.D.

Claim. A monopolist seller would select maximally differentiated qualities.

Consider what quality levels a monopolist offering two products with different identities would offer. We assume that the utilities for consumers match those presented in $\$ 2$. Suppose the monopolist can costlessly set each product's quality level to $q_{1} \equiv L \leq H \equiv q_{2}$ for any $q_{j} \in[q, \bar{q}]$ restricted to be similar (in the sense of Definition 1). Assume $\beta$ is high enough that the market is covered; that is, excluding any customers from buying two units is not profitable. In such a case, the price for the low-quality good must be at most $p_{L}=\beta$ to ensure that even consumers with $\theta=0$ buy two units. Assuming the market is covered, the monopolist's profits are $\Pi\left(p_{H}, p_{L}\right)=p_{H}\left(1-\left(p_{H}-p_{L}\right) /(H-\delta L)\right)+$ $p_{L}\left(1+\left(p_{H}-p_{L}\right) /(H-\delta L)\right)$, so the optimal price to charge for the high-quality good is $p_{H}^{*}\left(p_{L}\right)=p_{L}+(H-\delta L) / 2$. Plugging $p_{L}=\beta$ into the formula for $p_{H}^{*}(\beta)$ implies $\Pi^{*}(L, H)=$ $(H-\delta L) / 4+2 \beta$, which is maximized by selecting $L=q$ and $H=\bar{q}$. Note that a monopolist would maximize the difference in qualities even if the range of qualities available to firms were extended beyond the similar range as long as the market remained covered.

Proof of Proposition 5. Let $D=q(1-\delta)$ and restrict both firms to include the second unit for free with the first unit $(\lambda=0)$. The consumer behavior is simple: Everyone buys the cheaper two-unit bundle, and consumers with $\theta>\max \left(p_{i}\right) / D$ also buy the more expensive bundle. Suppose firm 1 charges $p_{1}$ for its two-unit bundle. Firm 2 can undercut $p_{1}$ slightly to obtain profit of $p_{1}$. Alternatively, firm 2 can charge a higher price (but still below $D$ ) and get monopoly demand of $1-p_{2} / D$ from the variety seekers. It follows that the best response of firm 2 to $p_{1}$ is to undercut when $p_{1} \geq D / 4$, and to charge $D / 4$ when $p_{1} \leq D / 4$. From symmetry of firms, there cannot be a pure-strategy equilibrium. We now construct a symmetric mixed-strategy equilibrium. Suppose firm 1 plays a continuous distribution $F$ on some interval $[A, B] \subseteq[0, D]$, and let $\Pi_{2}\left(p_{2}\right)$ be the expected profit of firm 2. For a symmetric equilibrium whereby firm 2 finds it optimal to also play $F$ on $[A, B]$, it must be that $B \geq D / 2$. Otherwise, firm 2 would benefit from deviating to higher prices because $\Pi_{2}^{\prime}(B)>0$ : For any price $p_{2} \geq B$, firm 2 is guaranteed to be the higher-priced firm, and get profit $p_{2}\left(1-p_{2} / D\right)$ which is increasing in price whenever $p_{2}<D / 2$. When firm 2 plays $B$, it charges the higher price almost surely, and thus collects profit $B[1-B / D]>0$. When firm 2 plays $A$, it charges the lower price almost surely, and thus collects profits $A$. From indifference, $A=$ $B[1-B / D]>0$. Now suppose firm 2 charges some price $p_{2}<D$. Then, $\Pi_{2}\left(p_{2}\right)=\int_{A}^{p_{2}} p_{2}\left[1-p_{2} / D\right] d F\left(p_{1}\right)+\int_{p_{2}}^{B} p_{2} d F\left(p_{1}\right)=$ $p_{2}-\left(p_{2}^{2} / D\right) F\left(p_{2}\right)$.

The indifference on $[A, B]$ implies $\Pi_{2}^{\prime}\left(p_{2}\right)=0$, which yields an ordinary differential equation: $D=2 p_{2} F\left(p_{2}\right)+$ $p_{2}^{2} f\left(p_{2}\right)=G^{\prime}(p)$ where $f=F$ and $G(p) \equiv p^{2} F(p)$ is a helpful reparametrization. The solution thus must satisfy $G(p)=$ $\int D d p-c=p D-c \Rightarrow F(p)=D / p-c / p^{2}$ for some constant $c$. $B$ fixes $c$ because: $1=F(B)=D / B-c / B^{2} \Rightarrow c=B(D-B)$, and so we can write $F(p)=D / p-(B(D-B)) / p^{2}$. This function is only a cumulative distribution function when its derivative $f$ is positive: $f(p)=F^{\prime}(p) \geq 0 \Leftrightarrow 2 B(D-B) \geq D p$ for all prices in the support. Specifically, it needs to be the case that $f(B)=(D-2 B) / B^{2} \geq 0 \Leftrightarrow B \leq D / 2$. Therefore (from above), the only candidate is $B=D / 2$ with $F(p)=\left(D /\left(4 p^{2}\right)\right)(4 p-D)$ and $f(p)=\left(D /\left(2 p^{3}\right)\right)(D-2 p)$, which is a valid distribution. The expected profit is the lower bound of the support: $A=$ $B[1-B / D]=D / 4$.

Now suppose firm 2 includes the second unit for free with the first unit $\left(\lambda_{2}=0\right)$ and plays according to the above $F$ while firm 1 is free to respond with an discount $\lambda_{1}$. First note that the effective bundle price $\tilde{p}_{1}=p_{1}\left(1+\lambda_{1}\right)$ of firm 1 cannot be outside of the $[D / 4, D / 2]$ support of $F$ : When $\tilde{p}_{1}>D / 2$, then firm 1 can never sell the second unit, and its profit is $\Pi_{1}=p_{1}\left(1-p_{1} / D\right)$ because consumers with $\theta>p_{1} / D$ choose variety. This profit is maximized at $\tilde{p}_{1}=D / 2$. Charging $\tilde{p}_{1}<D / 4$ cannot help either because everyone now buys both units. So raising the bundle price to $\tilde{p}_{1}=D / 4$ dominates. Now fix any $\tilde{p}_{1} \in[D / 4, D / 2]$ and consider varying $\lambda_{1}$. There are two cases, depending on the relative bundle prices:

Case $1\left(\tilde{p}_{1}<p_{2}\right)$. Consumers with $\theta>\left(p_{2}-\lambda_{1} p_{1}\right) /$ $(q(1-\delta))$ choose variety by buying just one unit of each firm, and consumers with $\theta<\left(p_{2}-\lambda_{1} p_{1}\right) /(q(1-\delta))$ buy two units of firm 1 product because it is the cheaper bundle. The profit of firm 1 is

$$
\begin{aligned}
\Pi_{1}\left(\tilde{p}_{1}, \lambda_{1}\right) & =p_{1}\left(1+\lambda_{1}\right) \frac{p_{2}-\lambda_{1} p_{1}}{q(1-\delta)}+p_{1}\left[1-\frac{p_{2}-\lambda_{1} p_{1}}{q(1-\delta)}\right] \\
& =\tilde{p}_{1}-\lambda_{1} p_{1}\left[1-\frac{p_{2}-\lambda_{1} p_{1}}{q(1-\delta)}\right],
\end{aligned}
$$

which obviously decreases in $\lambda_{1} p_{1}$ conditional on a constant bundle price $\tilde{p}_{1}$.

Case $2\left(\tilde{p}_{1}>p_{2}\right)$. No consumers buy the second unit of firm 1 , so the profit is $\Pi_{1}=p_{1}\left(1-p_{1} / D\right)$, which is increasing for all $p_{1}<D / 2$. So the firm wants as much as possible of the bundle price to be collected on the first unit, i.e., it wants to set $\lambda_{1}=0$.

Together, Cases 1 and 2 show that $\lambda_{1}=0$ dominates any $\lambda_{1}>0$ regardless of $p_{2}$. Therefore, the best response of firm 1 to firm 2's including the second unit for free with the first unit is to do the same with its own second unit. Q.E.D.

Proof of Proposition 6. First restrict both firms to include the second unit for free with the first unit $(\lambda=0)$. No consumers choose variety because buying the low-quality bundle in addition to the high-quality 
bundle involves an expense without any incremental benefit $(H+\delta H>H+L)$. Therefore, the competition is akin to a standard unit-demand duopoly with the difference in product qualities of $\Delta=(H-L)(1+\delta)$. The best-response functions are $p_{L}=p_{H} / 2, p_{H}=p_{L} / 2+\Delta$, the equilibrium prices are $p_{L}=\Delta / 3<p_{H}=2 \Delta / 3$, and the equilibrium profits are $\Pi_{L}=\Delta / 9$ and $\Pi_{H}=4 \Delta / 9$. The profits of both firms exceed the profits with unit pricing $(\lambda=1)$ because $4 R$ from the proof of Proposition 2 is smaller than $\Delta$ :

$$
\begin{gathered}
\Pi_{L}^{\text {unit }}=\frac{4}{9} R=\frac{4 C D}{9(D+C)}<\frac{(D+C)}{9}=\Pi_{L}^{\text {bundle }}, \\
\Pi_{H}^{\text {unit }}=\frac{16}{9} R=\frac{4 \cdot 4 C D}{9(D+C)}<\frac{4(D+C)}{9}=\Pi_{H}^{\text {bundle }},
\end{gathered}
$$

where $D=H-\delta L$ and $C=\delta H-L$.

Now allow each firm to use an second-unit discount $\lambda_{q}$. For clarity, reparametrize the strategies in terms of the 1st unit price as $p_{q}$ and the second-unit price by $r_{q} \equiv \lambda_{q} p_{q}$ under the $r_{q} \leq p_{q}$ constraint. The utility comparisons follow Equations $(1 \lambda),(2 \lambda)$, and $(3 \mathrm{D} \lambda)$, and consumer behavior either involves middle- $\theta$ consumers choosing variety whenever $\theta_{H}=\left(r_{H}-p_{L}\right) / C>\left(p_{H}-r_{L}\right) / D=\theta_{L}$ or it is identical to the above $\lambda=0$ case when $\theta_{H} \leq \theta_{L}$. Suppose $\theta_{H}>\theta_{L} \Rightarrow$ $\Pi_{L}\left(p_{L}, r_{L}\right)=p_{L}\left(\left(r_{H}-p_{L}\right) / C\right)+r_{L}\left(\left(p_{H}-r_{L}\right) / D\right)$. The $r_{q} \leq p_{q}$ constraint binds for the $L$ firm because its competition for the second unit $\left(p_{H}\right)$ is less intense than for the first unit $\left(r_{H}\right)$, and $L$ would always like to charge more for the second unit. Therefore, $L$ prefers unit pricing $\left(p_{0}=r_{0}\right)$ whenever some consumers choose variety. Given any $p_{0}=r_{0}$ and $\theta_{H}>$ $\theta_{L}$, the $H$ firm profit is $\Pi_{H}\left(p_{H}, r_{H}\right)=p_{H}\left(1-\left(p_{H}-p_{L}\right) / D\right)+$ $r_{H}\left(1-\left(r_{H}-p_{L}\right) / C\right)$. We now show that for any $r_{H}, p_{H}$ that support some consumers choosing variety, $H$ is better off charging $\varepsilon$ less for the second unit and $\varepsilon$ more for the first unit: $\theta_{H}>\theta_{L} \Leftrightarrow D r_{H}-C p_{H}>D p_{L}-C r_{L} \Rightarrow \partial \Pi_{H} / \partial p_{H}=$ $\left(p_{L}-2 p_{H}\right) / D>\left(p_{L}-2 r_{H}\right) / C=\partial \Pi_{H} / \partial r_{H}$. Therefore, sustaining variety-seeking behavior cannot be the best response of the high firm. Instead, it prefers to minimize $r_{H}$ at least until no consumers choose variety, beyond which point only the $r_{H}+p_{H}$ total influences profit. For example, $H$ can set $r_{H}=0$ and optimize: $\Pi_{H}^{\text {bundle }}\left(p_{H} \mid p_{L}\right)=p_{H}\left(1-\left(p_{H}-2 p_{L}\right) /(D+C)\right) \Rightarrow$ $p_{H}=p_{L}+(D+C) / 2$. The best response of the low-quality firm in turn cannot involve any consumers choosing variety because $r_{H}=0$ ensures the upper bound of the varietyseeking region is negative. Therefore, both firms charge the same total bundle prices $r_{q}+p_{q}$ and make the same profit as they would if they included the second unit for free with the first unit. Q.E.D.

\section{References}

Faison EWJ (1977) The neglected variety drive: A useful concept for consumer behavior. J. Consumer Res. 4(3):172-175.

Feinberg FM, Kahn BE, McAlister L (1992) Market share response when consumers seek variety. J. Marketing Res. 29(2):227-237.

Givon M (1984) Variety seeking through brand switching. Marketing Sci. 3(1):1-22.

Guo L (2006) Consumption flexibility, product configuration, and market competition. Marketing Sci. 25(2):116-130.

Kahn BE, Kalwani MU, Morrison DG (1986) Measuring varietyseeking and reinforcement behaviors using panel data. J. Marketing Res. 23(2):89-100.

Mazzeo M (2002) Product choice and oligopoly market structure. RAND J. Econom. 33(2):221-242.

McAlister L (1982) A dynamic attribute satiation model of varietyseeking behavior. J. Consumer Res. 9(3):141-150.

McAlister L, Pessemier EA (1982) Variety seeking behavior: An interdisciplinary review. J. Consumer Res. 9(3):311-322.

Moorthy KS (1988) Product and price competition in a duopoly. Marketing Sci. 7(2):141-168.

Raju PS (1980) Optimum stimulation level: Its relationship to personality, demographics, and exploratory behavior. J. Consumer Res. 7(3):272-282.

Ratner RK, Kahn BE, Kahneman D (1999) Choosing less-preferred experiences for the sake of variety. J. Consumer Res. 26(1):1-15.

Sajeesh S, Raju JS (2010) Positioning and pricing in a variety seeking market. Management Sci. 56(6):949-961.

Seetharaman PB, Che H (2009) Price competition in markets with consumer variety seeking. Marketing Sci. 28(3):516-525.

Shaked A, Sutton J (1982) Relaxing price competition through product differentiation. Rev. Econom. Stud. 49(1):3-13.

Trivedi M, Bass FM, Rao RC (1994) A model of stochastic varietyseeking. Marketing Sci. 13(3):274-297.

Villas-Boas M (2004) Consumer learning, brand loyalty, and competition. Marketing Sci. 23(1):134-145. 